\title{
卤素盐参与下有机电合成含氮杂环化合物的研究进展
}

\author{
周娅琴 $a$ 赵志恒 ${ }^{a} \quad$ 曾 亮 ${ }^{a}$ 李 鸣 ${ }^{a}$ 何永辉 $* a$ 谷利军 $*, a, b$ \\ $\left({ }^{a}\right.$ 云南民族大学民族医药学院 民族药资源化学教育部重点实验室 昆明 550025) \\ ( ${ }^{b}$ 贵州大学精细化工研究开发中心 绿色农药和农业生物工程教育部重点实验室 贵阳 550025)
}

\begin{abstract}
摘要 含氮杂环化合物广泛存在于医药、农药及天然产物中, 是许多具有生理活性的化合物和药物的基本骨架. 开发 高效、绿色的含氮杂环化合物的构建方法具有重要意义. 近年来, 卤素盐参与下有机电合成含氮杂环化合物取得了诸 多进展. 该类反应具有操作简单、绿色环保等特点. 综述了有机电化学合成反应中, 卤素盐作媒介, 含氮杂环化合物的 合成研究新进展.
\end{abstract}

关键词 卤素盐; 电化学合成; 含氮杂环

\section{Recent Advance in Organic Electrochemical Synthesis of Nitroge- nous Heterocyclic Compounds Involving Haloids as Mediators}

\author{
Zhou, Yaqin $^{a}$ \\ Zhao, Zhiheng $^{a}$ \\ Zeng, Liang ${ }^{a}$ \\ Li, Ming ${ }^{a}$ \\ He, Yonghui ${ }^{*, a}$ \\ $\mathrm{Gu}$, Lijun $*, a, b$ \\ $\left({ }^{a}\right.$ Key Laboratory of Chemistry in Ethnic Medicinal Resources, Ministry of Education, \\ School of Ethnic Medicine, Yunnan Minzu University, Kunmin 650500) \\ $\left({ }^{b}\right.$ Key Laboratory of Green Pesticide and Agricultural Bioengineering, Ministry of Education, \\ Guizhou University, Guivang 550025)
}

\begin{abstract}
Nitrogenous heterocyclic compounds are widely found in medicinal molecules, natural products and functional materials. Therefore, it has great significance to develop simple and efficient methods for the construction of these compounds. Recently, remarkable progress has been made in haloids mediated electrochemical synthesis of nitrogen heterocycles. Due to the relatively mild reaction condition and environmental protection, it provides a novel approach to construct nitrogen heterocycles. In this review, the recent developments in this area are summarized.

Keywords haloids; electrochemical synthesis; nitrogenous heterocyclic compounds
\end{abstract}

杂环化合物在自然界中普遍存在，天然的和合成的 杂环化合物在许多商业领域有重要和广泛的应用. 含氮 杂环类结构单元作为基础的杂环, 广泛存在于医药、农 药及天然产物中, 是许多具有生理活性的化合物和药物 的基本骨架 ${ }^{[1]}$. 此外, 它们还是合成某些功能材料的重 要原料. 因此, 发展合成含氮杂环化合物的方法受到了 化学家们的极大关注, 每年都有大量的文献、专利涉及 到它的合成研究 ${ }^{[2]}$.

有机电合成已成为有机合成中的一个重要组成部 分, 有机电合成利用电能驱动化学反应, 避免了化学氧 化剂或者还原剂的使用, 能从化学转化的源头上控制污 染, 具有绿色可持续、高效率以及高选择性等优点 ${ }^{[3]}$. 基 于这些优势, 有机电合成的发展突飞猛进, 许多结构新
颖的含氮杂环化合物相继被合成出来 ${ }^{[4]}$. 最近在卤素盐 参与下, 有机电合成含氮杂环化合物取得了较大进展 ${ }^{[5]}$ 利用电化学方法可以有效地实现底物或卤素离子在阳 极上的氧化，形成高活性的反应中间体，是构建含氮杂 环化合物的一种绿色合成方法. 本文在前人研究报道的 基础上, 同时结合本课题组的工作, 综述了有机电化学 合成反应中, 卤素盐作媒介, 含氮杂环化合物的合成研 究新进展.

\section{1 碘盐作媒介参与反应}

\section{1 三元含氮杂环化合物的合成}

三元含氮杂环呋丙啶是一种重要的化工原料. 2015 年, 曾程初课题组 ${ }^{[6]}$ 利用玻璃碳作阳极, 铁板作阴极,

\footnotetext{
*Corresponding authors. E-mail: gulijun2005@126.com, hee_csu@126.com

Received July 21, 2020; revised August 29, 2020; published online September 22, 2020.

国家自然科学基金(No. 21662045)、贵州大学绿色农药实验室开放基金(No. 2018GDGP0103)资助项目。
} 
在四丁基碘化铵参与下, 实现了 $N$-氨基邻苯二甲酰亚 胺和苯乙烯有机电合成听丙啶衍生物, 降低反应温度有 利于增加反应收率. 优化条件对各种取代的苯乙烯表现 出较好的适应性, 然而, 脂肪环状的烯烃底物反应性差. 可能的反应过程如 Scheme 1 所示: 首先, 四丁基碘化铵 中的碘离子在阳极上被氧化为单质碘, 随后和四丁基碘 化铵得到 $n-\mathrm{Bu}_{4} \mathrm{NI}_{3}$ 复合物. 在可见光的作用下, 该复合 物分解为 $\mathrm{I} \bullet$ 及 $n \mathrm{Bu}_{4} \mathrm{NI}_{2} \bullet$. 在 $\mathrm{I} \bullet$ 的作用下, $N$-氨基邻苯二甲 酰亚胺生成氨基自由基, 其进一步与烯烃加成得中间体 A. 最后经由夺氢、环化得目标产物.

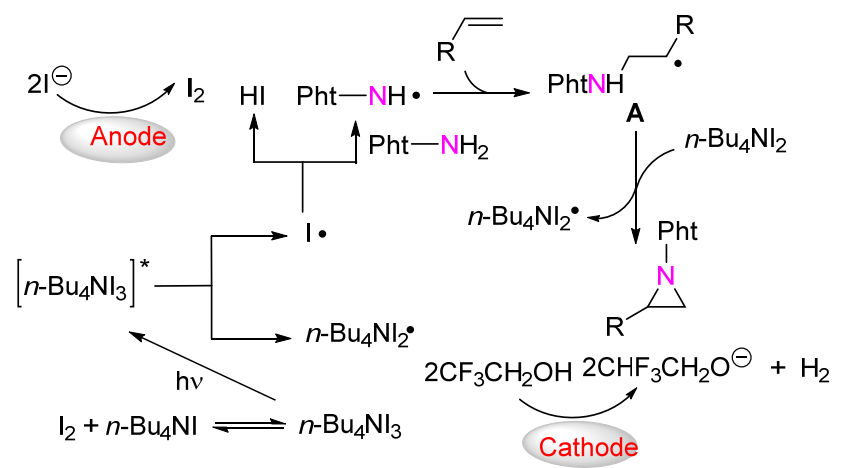

图式 1 碘盐为媒介电化学催化合成叫丙啶的可能机理

Scheme 1 Proposed reaction mechanism for electro-catalyzed synthesis of aziridines mediated by iodized salt

2019 年, Tan 课题组 ${ }^{[7]}$ 利用电化学诱导碘盐催化策 略, 在无金属和无外部氧化剂条件下实现了 $\beta$-氨基酮的 $\mathrm{C}\left(\mathrm{sp}^{3}\right)-\mathrm{H} / \mathrm{N}-\mathrm{H}$ 脱氢偶联反应, 合成了系列唤丙啶衍 生物, 最高收率可达 $87 \%$ (Scheme 2). 该反应有很好的 官能团兼容性及选择性.

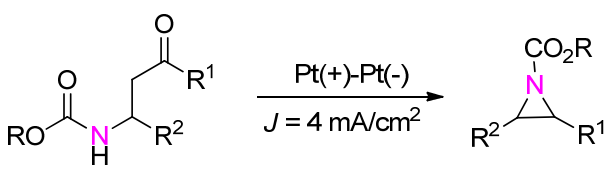<smiles>CCOC(=O)N1C(C(=O)c2ccccc2)C1c1ccccc1</smiles>

$83 \%$<smiles>CCOC(=O)N1C(C(=O)c2ccc(Cl)cc2)C1c1ccccc1</smiles>

$64 \%$<smiles>CCOC(=O)N1C(C(=O)c2ccco2)C1c1ccccc1</smiles>

$59 \%$
图式 2 电化学催化 $\beta$-氨基酮合成呋丙啶衍生物 Scheme 2 Electrochemical synthesis of aziridines from $\beta$-amino ketones

\section{2 五元含氮杂环化合物的合成}

1,3,4-惡二唑是一类非常重要的五元含氮含氧杂环 化合物，广泛应用于医药、农药等领域. 2013 年, Wang 课题组 ${ }^{[8]}$ 率先报道了取代 1,3,4-噁二唑的合成, 以甲醇 为反应溶剂, 氢氧化钾作碱, 在碘化钾的参与下多种官 能团取代的 1,3,4-啞二唑均能顺利合成, 最高收率可达
79\%（Scheme 3). 通过控制实验，作者推测苯甲酰肼先 氧化为苯甲醛，随后和另一分子苯甲酰肼缩合得酰腙中 间体，最后通过环化、脱氢得到目标产物取代 1,3,4-啞 二唑.<smiles>COc1cc([Al]C(=O)NN)ccc1Cl</smiles><smiles>Cc1ccc(-c2nnc(-c3ccc(C)cc3)o2)cc1</smiles><smiles>Cc1cccc(-c2nnc(-c3cccc(C)c3)o2)c1</smiles><smiles></smiles><smiles>CC(C)(C)OC(=O)c1cccs1</smiles>

图式 3 电化学催化合成啞二唑化合物

Scheme 3 Electrosynthesis of oxadiazoles

1,2,4-噻二唑及 1,2,3-噻二唑衍生物作为一种含有多 个杂原子的五元含氮杂环类化合物，具有抗炎、抗菌等 生物活性, 还是一种重要的农药活性分子. 2018 年, 唐 海涛课题组 ${ }^{[9]}$ 以硫代酰胺为原料, 甲醇和乙腈混合溶剂 为反应溶剂, 在碘化铵的参与下合成了系列 3,5-二取代 的 1,2,4-噻二唑，克级反应收率可达 $88 \%$ (Scheme 4). 控 制实验结果显示芳基取代的硫代酰胺反应活性高于烷 基的硫代酰胺.
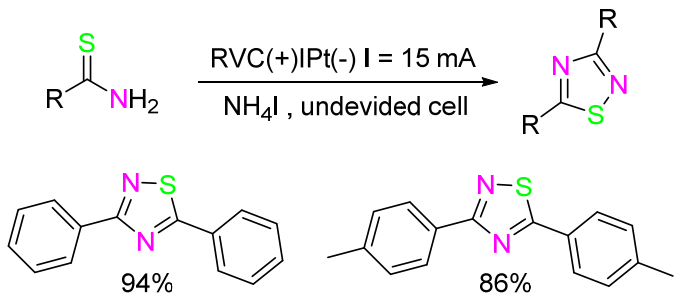<smiles>CC(C)(C)c1nsc(C(C)(C)C)n1</smiles>

$70 \%$<smiles>Clc1cccc(-c2nsc(-c3cccc(Cl)c3)n2)c1</smiles>

$79 \%$
图式 4 电化学催化合成 1,2,4-噻二唑

Scheme 4 Electrochemical synthesis of 1,2,4-thiadiazoles

2019 年, 该课题组 ${ }^{[10]}$ 实现了无金属、无氧化剂条件 下有机电合成单取代 1,2,3-噻二唑衍生物. 进一步的反 应底物拓展结果显示，该反应适用范围较窄，产物限于 芳(杂芳)基取代的 1,2,3-噻二唑，没有实现二取代及 4烷基取代的 1,2,3-噻二唑合成. 通过控制实验，作者提 出如 Scheme 5 所示的反应机理: 碘负离子在阳极失去 电子氧化为单质碘，随后和 $N$-磺酰基酰腙反应生成 $\alpha$ 碘代物，其经由消除、加成、环化等步骤得到 4-取代的 1,2,3-噻二唑.

2018 年, Zhang 课题组 ${ }^{[11]}$ 以 $\beta$-胺基芳香酮为原料, 


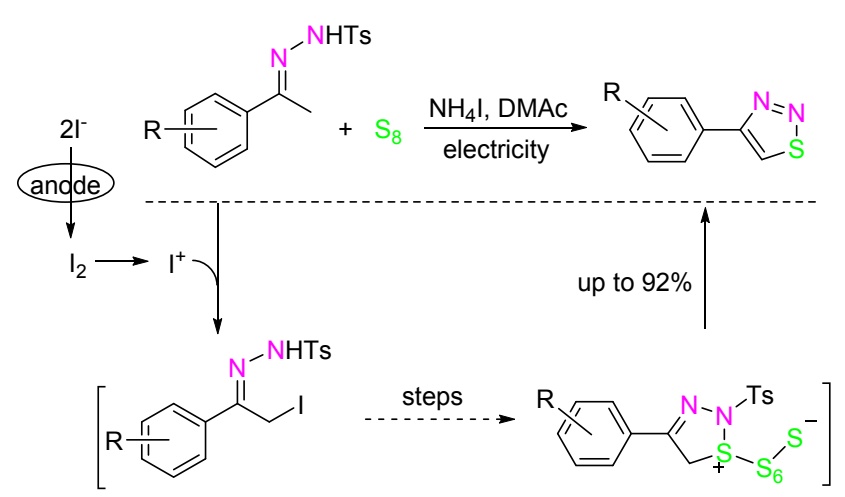

图式 5 电化学催化合成 1,2,3-噻二唑的可能机理

Scheme 5 Proposed reaction mechanism for electro-catalyzed synthesis of 1,2,3-thiadiazoles

利用碘化钾作电解质和媒介, 在无隔膜电解池中, 室温 下合成了系列 2-噁唑啉衍生物(Scheme 6). 克级反应收 率仍然可达 69\%.

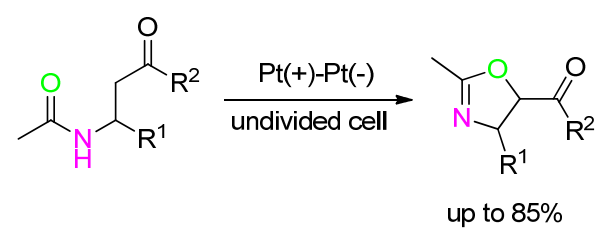

图式 6 电化学合成三取代的 2-噁唑啉衍生物

Scheme 6 Electrosynthesis of trisubstituted 2-oxazolines

二氧化碳作为空气中的重要组分，廉价易得、无毒、 稳定、可再生循环使用. 2016 年, Yuan 课题组 ${ }^{[12]}$ 以 NaI 为媒质, 实现了苯肼、多聚甲醛及二氧化碳的三组分反 应合成了系列噁二唑酮类化合物(Scheme 7). 在最优反 应条件下多种取代的苯肼能够顺利完成环化反应，反应 具有良好的底物适应性. 机理研究显示, 碘负离子首先

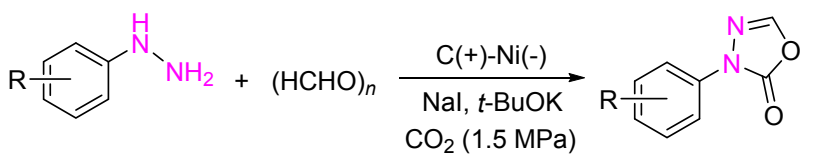<smiles>O=c1ocnn1-c1cccc(Br)c1</smiles><smiles>O=c1ocnn1-c1ccc(Cl)cc1</smiles><smiles>N#Cc1ccc(-n2ncoc2=O)cc1</smiles><smiles>COc1ccc(-n2ncoc2=O)cc1</smiles>

图式 7 二氧化碳、苯肼和多聚甲醛的电化学合成二唑酮类化 合物

Scheme 7 Electrochemical conversion of $\mathrm{CO}_{2}$ with aryl hydrazines and paraformaldehyde into 1,3,4-oxadiazol-2(3H)-one derivatives
在阳极上被氧化为单质碘，随后参与反应。

多组分串联反应具有原子经济性和步骤经济性等 优点. 2018 年，该课题组 ${ }^{[13]}$ 实现了苯肼、多聚甲醛、醋 酸铵及醇的四组分环化反应合成 1,5 -二取代的三氮唑衍 生物. 当反应不加入醇, 产物为 1-芳基-1,2,4-三氮唑衍 生物(Scheme 8). 控制实验显示反应为自由基反应历程. 反应机理研究显示, 四丁基碘化铵中的碘离子在阳极上 被氧化为单质碘.

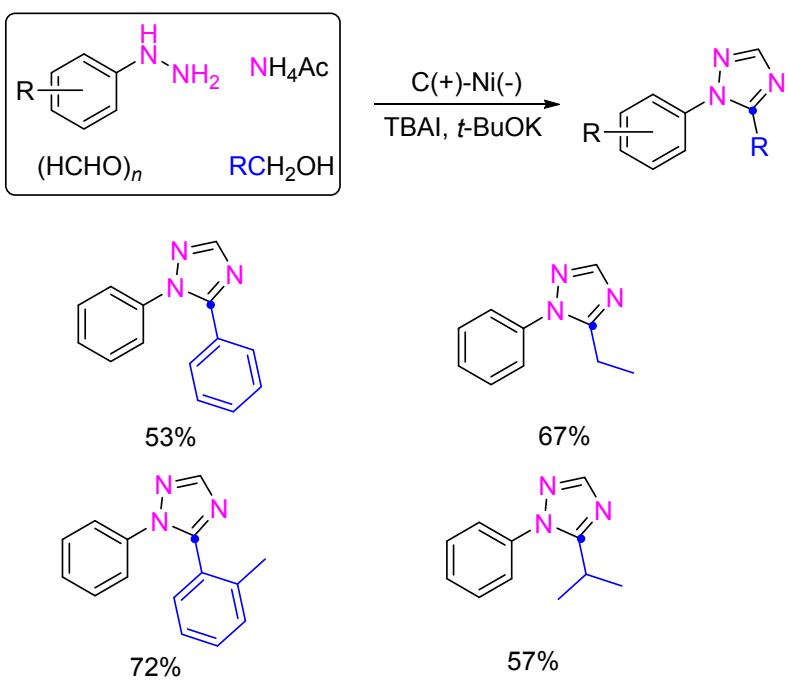

图式 8 多组分电化学催化合成合成 1,5-二取代的三氮唑衍生 物

Scheme 8 Multicomponent electro-catalyzed synthesis of 1,5disubstituted triazoles

最近, 本课题组 ${ }^{[14]}$ 以 $\mathrm{NH}_{4} \mathrm{I}$ 为媒质, 室温下实现了 芳香甲基酮和胺电化学脱氢 $[2+2+1]$ 环化反应合成咪 唑(Scheme 9). 当使用乙腈作反应溶剂时, 反应效果最 好，目标产物的收率可达 85\%. 烯基取代、炔基取代的 胺均能得到目标产物. 将反应规模由毫克级别放大到克 级别, 以 $9 \mathrm{~mA}$ 的恒电流电解 $10 \mathrm{~h}$, 能以 $74 \%$ 的分离收 率得到相应的咪唑.

\section{3 苯并含氮杂环化合物的合成}

合成苯并噁唑简单有效的方法是氧化邻亚胺基酚 席夫碱中的 $\mathrm{C}-\mathrm{H}$ 键和 $\mathrm{O}-\mathrm{H}$ 键, 然而反应往往需要加 入氧化剂, 如过氧化物、高价碘试剂等. 2013 年, 曾程初 课题组 ${ }^{[15]}$ 在不加外部氧化剂及过渡金属的条件下，电 化学氧化邻亚胺基酚席夫碱中的 $\mathrm{C}-\mathrm{H}$ 键和 $\mathrm{O}-\mathrm{H}$ 键合 成苯并噁坐，借助于两相反应系统，以 $\mathrm{NaI}$ 为媒介，合

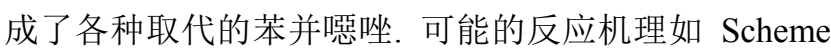
10 所示: 水相中碘负离子在阳极首先被氧化为单质碘, 在 $\mathrm{Na}_{2} \mathrm{CO}_{3} / \mathrm{NaHCO}_{3}$ 组成的缓冲溶液中, 单质碘生成了 氧化能力强的 $\mathrm{IO}^{-}$、 $\mathrm{IO}^{2-}$ 等, 随后进入有机相氧化 2,3二氢苯并噁唑中间体. 


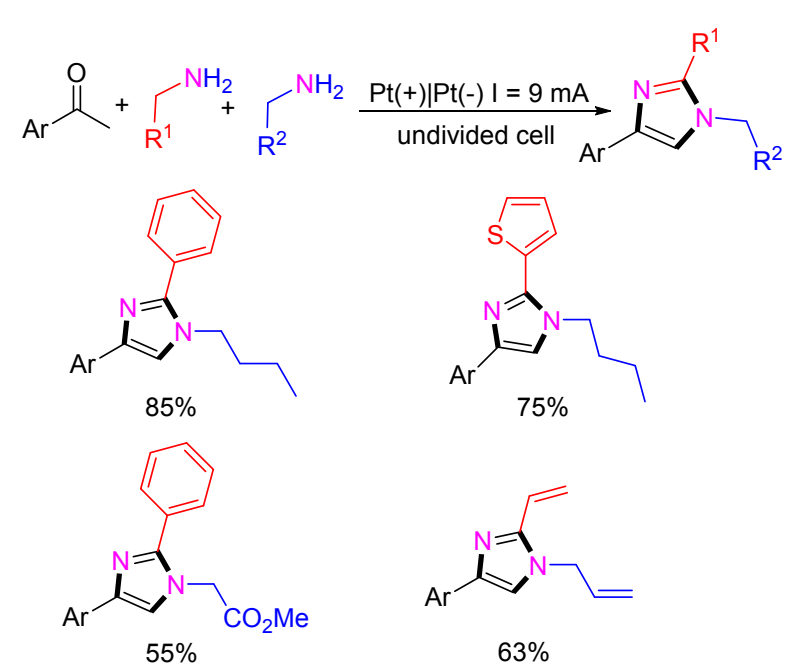

图式 9 碘盐为媒介电化学催化合成取代咪唑

Scheme 9 Electro-catalyzed synthesis of substituted imidazoles mediated by iodized salt

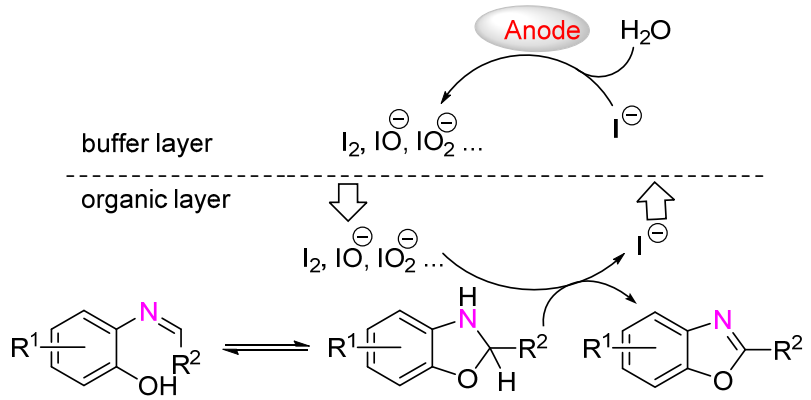

图式 10 碘盐为媒介电化学催化合成苯并噁唑的可能机理

Scheme 10 Proposed reaction mechanism for electro-catalyzed synthesis of benzoxazoles mediated by iodized salt

交叉脱氢偶联反应具有高原子经济性, 是构建化学 键的有效方法, 但该反应需要化学计量的氧化剂或贵金 属作为催化剂. 2014 年, 曾程初课题组 ${ }^{[16]}$ 通过电化学方 法在无金属和氧化剂参与条件下, 实现了苯并噁唑和脂 肪胺的交叉脱氢偶联反应(Scheme 11). 在优化的反应条 件下，作者研究了 2-氨基取代的苯并噁唑的合成. 苯并 噁唑环上的苯环不同位置含有不同取代基的底物，均可 以高收率得到 2-氨基苯并噁唑产物，而引入强吸电子基 团 $\left(\mathrm{NO}_{2}\right)$ 后收率降低. 反应的机理研究显示, 在醋酸的 作用下, 胺和苯并噁坐加成得到 2,3-二氢苯并噁唑中间 体，随后被 $\mathrm{I}^{+}$氧化得到 2-氨基苯并噁唑.

含氮杂环吲哚具有特殊的化学性质和生理活性, 关 于吲哚及其衍生物的化学合成研究和报道层出不穷. 2017 年, 雷爱文课题组 ${ }^{[17]}$ 以 $N$-苯基烯胺为原料, 碘化 钾作媒质, 采用单室电解槽和恒电流电解, 无需过渡金 属催化剂和氧化试剂, 实现了 $N$-苯基烯胺分子内 $\mathrm{C}\left(\mathrm{sp}^{2}\right)-\mathrm{H} / \mathrm{C}\left(\mathrm{sp}^{2}\right)-\mathrm{H}$ 脱氢偶联反应, 顺利地合成了系 列吲哚衍生物. 通过底物调控还可以构建咪唑 $[1,2-a]$ 并

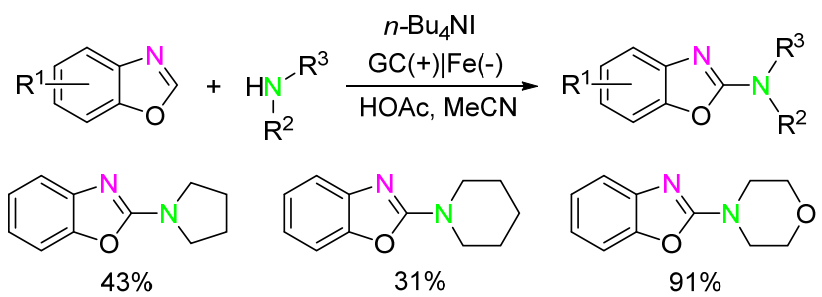

图式 11 电化学催化合成 2-氨基苯并噁唑

Scheme 11 Electro-catalyzed synthesis of 2-aminobenzoxazoles 吡啶杂环化合物. 控制实验显示反应为自由基反应历 程. 可能的反应机理如 Scheme 12 所示: 碘负离子在阳 极首先被氧化为 $\mathrm{I}^{+}$, 接着和 $N$-苯基烯胺反应得到 $N$-碘 代烯胺， $N$-碘代烯胺均裂得到氮自由基中间体，随后经 过结构互变、环化、脱氢等步骤得到目标化合物吲哚.

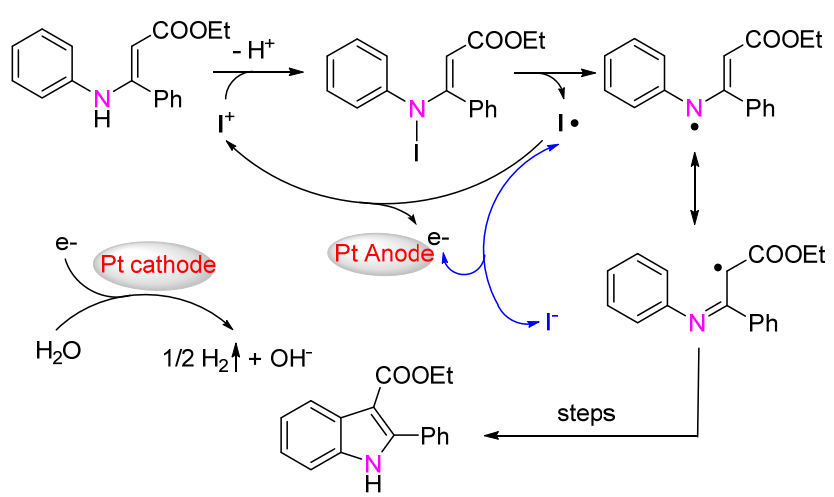

图式 12 碘盐媒介电化学催化 $N$-苯基烯胺合成吲哚的可能机 理

Scheme 12 Proposed reaction mechanism for electro-catalyzed synthesis of substituted indoles mediated by iodides

2018 年, 汪志勇课题组 ${ }^{[18]}$ 利用电化学诱导碘盐催 化策略，实现了 2-甲基含氮杂环和芐胺或氨基酸分子间 的 $\mathrm{C}\left(\mathrm{sp}^{3}\right)-\mathrm{H} / \mathrm{N}-\mathrm{H}$ 脱氢交叉偶联反应合成咪唑并含氮 杂环(Scheme 13). 反应机理的研究显示, 碘负离子首先 被氧化形成分子碘，接着与 2-甲基喹啉的异构体反应生 成 2-(碘甲基)喹啉，随后，经亲核取代、氧化、分子内胺 化、芳构化等过程得到咪唑并含氮杂环化合物. 紧接着, 他们通过加入 TMSCN, 实现了电催化的三组分反应合 成 3-氧基取代的咪唑并含氮杂环. 反应条件温和, 底物 普适性广. 值得指出的是, 该反应属首次在非金属条件 下合成了 3-氧基取代咪唑并含氮杂环, 为该类化合物的 合成提供了一种简单绿色的方法. 2019 年, 该课题组 ${ }^{[19]}$ 在前期工作的基础上，通过底物巧妙的设计，高效地合 成了系列 1,3-二取代的咪唑[1,5-a]并喹啉化合物.

咪唑 $[1,2-a]$ 并吡啶广泛应用于医药、农药领域. 大 量研究表明该类化合物具有较好的生物活性，如杀菌、 抗病毒、抗癌等. 它的合成方法一直受到人们广泛的关 注. 2019 年, Chen 课题组 ${ }^{[20]}$ 以廉价易得的邻胺基吡啶和 苯乙酮为原料, 在无金属和无外部氧化剂条件下，以氢 


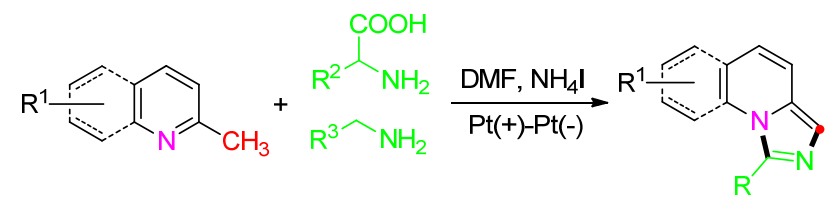

up to $96 \%$

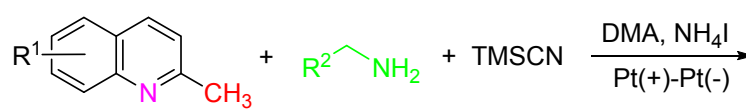<smiles>[R][Y]1ccc2ccc3c(C#N)nc([R])n3c2c1</smiles><smiles>N#Cc1nc(-c2ccc(F)cc2)n2c1ccc1ccccc12</smiles>

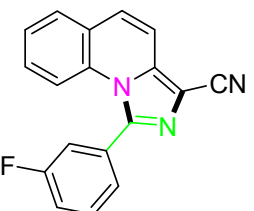

$98 \%$ $88 \%$

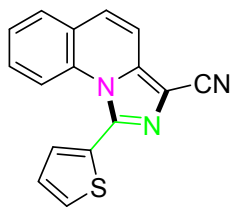

$56 \%$

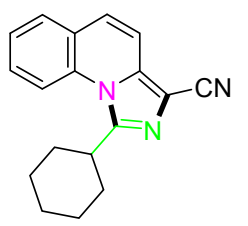

$63 \%$
图式 13 电化学催化合成取代的咪唑并含氮杂环

Scheme 13 Electro-catalyzed synthesis of substituted imidazo-fused $N$-heterocycles

碘酸为媒质, 乙醇为反应溶剂, 各种甲基酮均能通过 $\mathrm{C}-\mathrm{N}$ 键和 $\mathrm{C}-\mathrm{C}$ 键的构建合成系列咪唑 $[1,2-a]$ 并吡啶 (Scheme 14). 当加入二苯二硫醚或二苯二硒醚时, 可以 实现酮、胺、硫(硒)醚三组分串联反应.

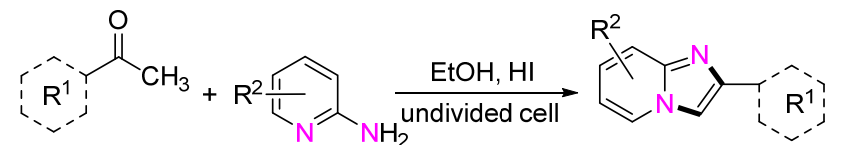

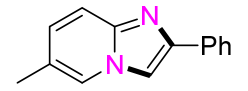

$73 \%$<smiles>Cc1ccn2cc(-c3ccccc3)nc2c1</smiles>

$75 \%$

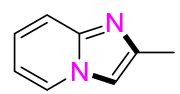

$69 \%$

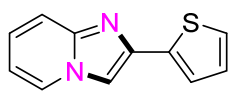

$57 \%$
图式 14 电化学催化合成咪唑 $[1,2-a]$ 并吡啶

Scheme 14 Electro-catalyzed synthesis of imidazo[1,2-a]pyridines

\section{4 其它氮杂环化合物的合成}

吲哚-2,3-二酮具有抗炎、抗菌等生理活性, 一直是 有机合成化学家的研究内容之一. 2017 年汪志勇课题 组 ${ }^{[21]}$ 利用电化学诱导碘盐催化策略, 在不加金属及过
氧化物的条件下，实现了邻 $\mathrm{N}$ 取代的氨基苯乙酮一步构 建吲哚-2,3-二酮。该反应过程涉及到三个 $\mathrm{C}\left(\mathrm{sp}^{3}\right)-\mathrm{H}$ 键、 一个 $\mathrm{N}-\mathrm{H}$ 键和一个 $\mathrm{O}-\mathrm{O}$ 键的断裂 (Scheme 15). 反应 的机理研究显示, 反应首先经过四丁基碘化铵中的碘离 子在阳极上被氧化为碘自由基，随后经过夺氢、氧气插 入等反应步骤合成目标化合物. 底物拓展结果显示, $\mathrm{N}$ 取代基为酰基时，几乎无产物生成.

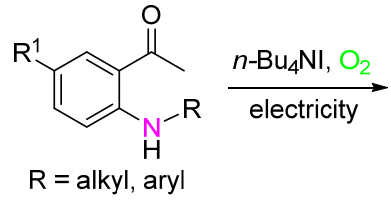<smiles>[R16]c1ccc2c(c1)C(=O)C(=O)N2[R16]([R16])([H])[H]</smiles>

图式 15 电催化氨基取代苯乙酮合成吲哚-2,3-二酮 Scheme 15 Electrocatalytic $\mathrm{C}-\mathrm{H} / \mathrm{N}-\mathrm{H}$ coupling of 2'-aminoacetophenones for the synthesis of isatins

2016 年，曾程初课题组 ${ }^{[22]}$ 以四丁基碘化铵为媒质， 甲醇作反应溶剂，实现了邻氨基苯乙烯分子内胺氧化反 应合成含氮杂环吲哚啉(Scheme 16). 条件优化结果显 示，氯盐、溴盐作反应媒质均无目标产物. 基于 NMR 对反应进行监测的结果，作者提出反应可能经过环碘鎓 离子过渡态. 当反应规模由毫克级别放大到克级别，仍 能以 $68 \%$ 的分离收率得到相应的吲哚啉，展现了该方法 潜在的工业应用前景.
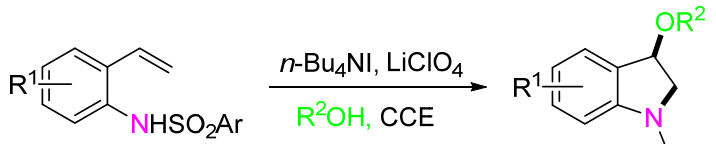

up to $80 \% \mathrm{SO}_{2} \mathrm{Ar}$

图式 16 电催化邻氨基苯乙烯分子内胺氧化合成含氮杂环吲 哚啉

Scheme 16 Electrochemically catalyzed amino-oxygenation of styrenes for the synthesis of indolines

吡嗪类化合物良好的生物活性以及结构易于修饰 的特点，在医药、农药、材料等众多领域显示出巨大的 应用潜力. 2019 年雷爱文课题组 ${ }^{[23]}$ 在电化学条件下，以 廉价易得的酮和二胺为原料, 合成了系列吡嗪衍生物 (Scheme 17). 反应条件优化结果显示: 反应在不加入 Brønsted 酸的情况下，收率仅 $23 \%$. 当加入催化量的 Brønsted 酸对甲苯磺酸后，收率可达 $83 \%$. 值得一提的 是，反应在空气中仍能顺利发生. 最优反应条件对氯、 溴等卤素，烷基、三氟甲基、烷氧基、腈基等都有很好 的兼容性.

吡啶 $[1,2-a]$ 并苯并咪唑是一类重要的含氮杂环化合 物, 大量研究表明该类化合物具有较好的生物活性, 如 杀虫、杀菌、除草、抗病毒、抗癌等. 过渡金属或高价 碘试剂催化下的 $\mathrm{C}-\mathrm{H}$ 氨化反应是构建该杂环常用的方 


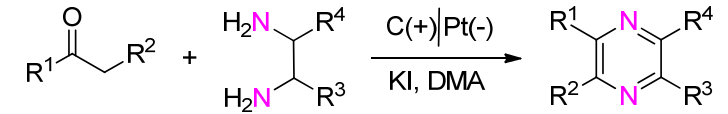<smiles>Cc1nccnc1Cl</smiles>

$\mathrm{NC}$<smiles>c1ccc(-c2cnccn2)cc1</smiles>

$51 \%$<smiles>c1cnc2c(n1)CCCCC2</smiles>

$50 \%$<smiles>Cc1nc2c(nc1Cl)CCCC2</smiles>

$74 \%$
图式 17 电化学催化 $[4+2]$ 成环合成吡嗪衍生物

Scheme 17 Electrochemical catalysis leads to [4+2] annulation for the synthesis of pyrazines

法. 最近陈建宾课题组 ${ }^{[24]}$ 独辟蹊径, 以邻苯氨基吡啶为 原料, 碘化钠为媒质, 在不加过渡金属及高价碘试剂的 条件下, 合成了系列吡啶 [1,2- $a$ ]并苯并咪唑衍生物 (Scheme 18). 有趣的是个别化合物表现出良好的光化 学性质, 具有较大的斯托克斯位移, 已成功应用于亚细 胞成像. 随后通过改变电极材料实现电流密度的调控, 进一步合成了四芳基肼骨架衍生物. 通过密度泛函理论 计算揭示了可能的反应途径.

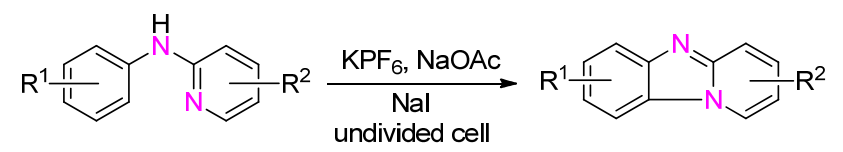<smiles>Fc1ccc2nc3ccc(Cl)cc3n2c1</smiles><smiles>Cc1ccc2nc3ccc(F)cn3c2c1</smiles><smiles>O=[N+]([O-])c1cccc(F)c1</smiles><smiles></smiles>
$50 \%, 0.59 g$<smiles>COc1ccc2nc3ccc(F)cn3c2c1</smiles><smiles>Fc1ccc2nc3cc(Cl)c(F)cn3c2c1</smiles>

图式 18 电化学催化合成吡啶[1,2-a]并苯并咪唑衍生物 Scheme 18 Electro-catalyzed synthesis of pyrido[1,2-a]-benzimidazoles

\section{2 溴盐作媒介参与反应}

相较于碘盐, 溴盐作为媒介参与含氮杂环化合物的 合成文献较少. 2016 年, 曾程初课题组 ${ }^{[25]}$ 使用催化量的 $\mathrm{NH}_{4} \mathrm{Br}$ ，以芳基亚磺酸钠为自由基源，1,4-二氧六环/水 作溶剂, 在电化学条件下原位产生磺酰基自由基, 实现 了丙烯酰胺的双官能团化反应，一步合成含磺酰基官能 团的2-吲哚啉酮衍生物(Scheme 19). 脂肪族的亚磺酸钠 也具有很好的兼容性. 随后作者尝试一锅法合成目标化 合物， $N$-甲基、 $N$-苯基、 $N$-茮基苯胺均能得到中等产率 的 2-吲哚啉酮.

最近, 该课题组 ${ }^{[26]}$ 通过反应条件的调控, 实现了含 三氟甲基官能团的 2-吲哚啉酮的合成. 该反应以乙腈为

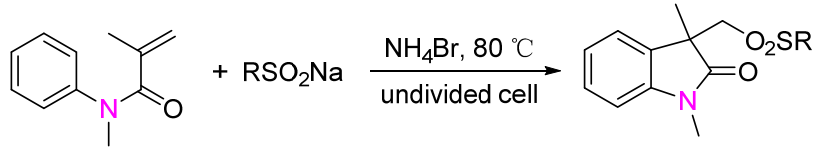<smiles>CN1C(=O)C(C)(CS(=O)(=O)c2ccccc2)c2ccccc21</smiles>

$55 \%$

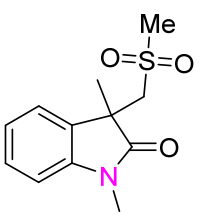

$85 \%$<smiles>CN1C(=O)C(C)(CS(=O)(=O)c2ccc(Cl)cc2)c2ccccc21</smiles>

$69 \%$
图式 19 溴盐为媒介电化学催化合成 2-吲哚啉酮衍生物 Scheme 19 Electro-catalyzed synthesis of oxindoles mediated by bromides salt.

溶剂, 四丁基溴化铵作为媒介, $75{ }^{\circ} \mathrm{C}$ 下无隔膜电解池中 电解，目标产物分离产率可达 $90 \%$. 当反应用水相溶液 时, 产率仅为 $52 \%$, 机理研究表明, 电化学条件下产生 的三氟甲磺酰基溴在阴极还原生成三氟甲基自由基，然 后和丙烯酰胺中的碳碳双键加成，关环得到目标产物 (Scheme 20).

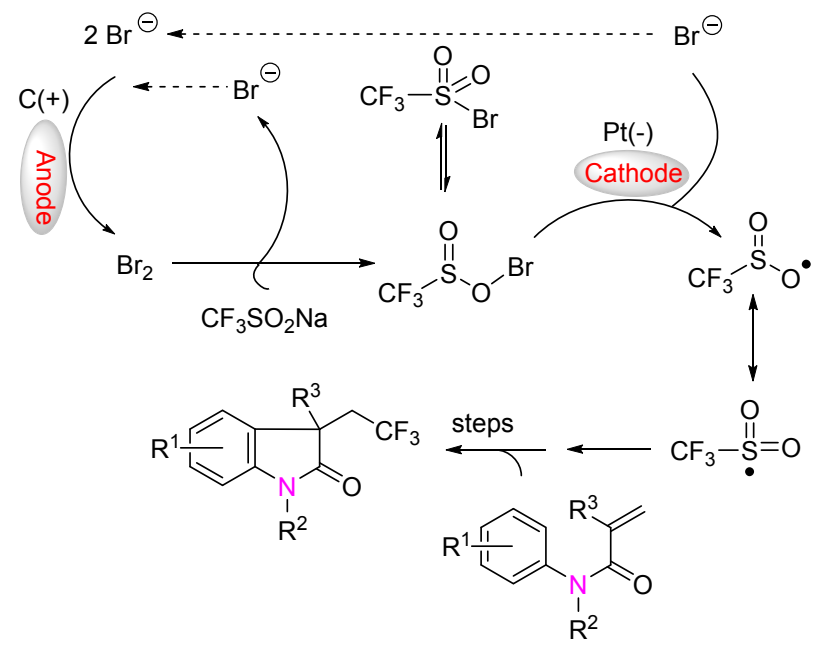

图式 20 溴盐为媒介电化学催化合成含三氟甲基官能团的 2吲哚啉酮的可能机理

Scheme 20 Proposed reaction mechanism for electro-catalyzed trifluoromethylation/cyclization of $\mathrm{N}$-arylacrylamides mediated by bromides salt

氮中心自由基是有机合成中的一类重要活性中间 体，在 $\mathrm{C}-\mathrm{N}$ 键的构建以及含氮杂环化合物的合成中具 有不可忽视的作用. 一般地, 氮中心自由基主要通过 $\mathrm{N}-\mathrm{X}$ 键的断裂实现. 2018 年, 曾程初课题组 ${ }^{[27]}$ 以 $\mathrm{NaBr}$ 为媒介, 电流密度 $8.9 \mathrm{~mA} / \mathrm{cm}^{2}$, 恒电流电解 $2 \sim 3 \mathrm{~h}$, 室 温下合成了系列内酰胺化合物. 该反应有很好的官能团 兼容性及选择性, 副产物只产生氢气，具有高效、绿色 等特点. 当反应放大到克级规模时，其分离产率仍能达 到 79\%，展现了该方法潜在的应用前景.

2019 年, Rueping 课题组 ${ }^{[28]}$ 用 $\mathrm{KBr}$ 为媒介, $N$-磺酰基 脂肪胺为原料, 在以石墨碳为阳极、不锈钢 316 为阴极 
构成的无隔膜电解槽中进行电解, 以 $29 \%$ ～ $82 \%$ 的产率 得到各种吡咯烷衍生物(Scheme 21). 在最优反应条件 下，作者利用流式反应器进行放大实验，产率达 $76 \%$. 通过控制实验，他们提出了两种可能的反应机理：一种 是溴负离子在阳极失去电子氧化为单质溴, 另一种是醇 氧负离子在阳极失去电子氧化为醇氧自由基.

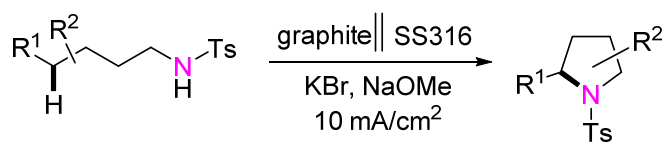<smiles>CCC1(C)CC(c2ccccc2)N([13CH3])C1</smiles>

$75 \%$
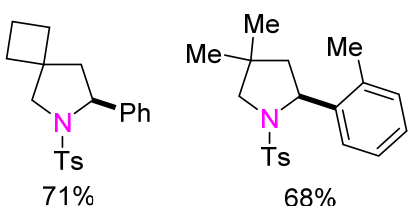

$68 \%$
图式 21 澳盐为媒介电化学催化合成吡咯烷衍生物 Scheme 21 Electro-catalyzed synthesis of pyrrolidines mediated by bromides salt.

2019 年, Zhang 课题组 ${ }^{[29]}$ 以 $N$-(2-吡啶基)酰胺为原 料, 四丁基溴化铵作媒质, 石墨作为阳极, 铂作为阴极, 在 $7 \mathrm{~mA}$ 恒定电流作用下分离得到目标产物 1,2,4-三氮 唑 $[1,5-a$ 吡啶 $(92 \%)$, 作者还笁选了其他反应条件, 并未 获得更好的结果. 在最优反应条件下, 卤素、烷基、三 氟甲基、烷氧基等都有很好的适应性. 放大实验仍能获 得 $82 \%$ 的分离产率. 通过循环伏安法实验, 作者提出两 种可能的反应机理(Scheme 22): 一种是通过 $\mathrm{N}-\mathrm{Br}$ 键 的断裂产生的氮中心自由基机理(path a), 另一种是直 接溴化亚胺的 $\mathrm{N}-\mathrm{H}$ 键 (path b).

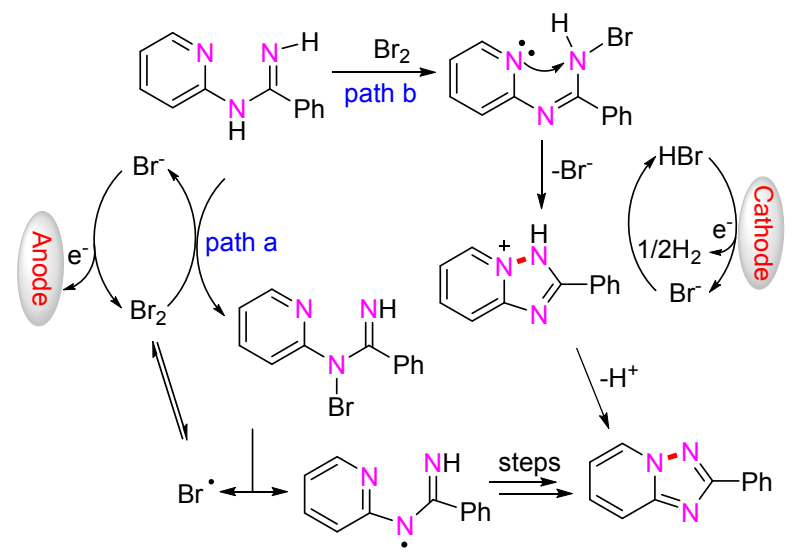

图式 22 溴盐为媒介电化学催化合成 $1,2,4$-三氮唑 $[1,5-a]$ 吡啶 的可能机理

Scheme 22 Proposed reaction mechanism for electro-catalyzed synthesis of 1,2,4-triazole[1,5-a]pyridine mediated by bromides salt

\section{3 氟盐作媒介参与反应}

氟盐作为媒介参与含氮杂环化合物的合成通常需
要芳基碘化物. 电解质 $\mathrm{Et}_{3} \mathrm{~N}-5 \mathrm{HF}$ 性能优于 $\mathrm{Et}_{3} \mathrm{~N}-3 \mathrm{HF}$, 对甲基碘苯在 $\mathrm{Et}_{3} \mathrm{~N}-5 \mathrm{HF}$ 存在下的阳极氧化能够生成二 氟碘苯. 基于这一策略, 2019 年 Waldvogel 课题组 ${ }^{[30]}$ 以 $\mathrm{Et}_{3} \mathrm{~N}-5 \mathrm{HF}$ 作为媒介, 烯丙基酰胺为原料, 通过电化学方 法在无金属和氧化剂参与条件下，合成了系列 5-氟甲 基-2-噁唑啉类化合物 (Scheme 23). 当电流密度 8.9 $\mathrm{mA} / \mathrm{cm}^{2}$, 恒电流电解 $15 \mathrm{~h}$, 产率可达 $95 \%$. 可能反应机 理为: 在 $\mathrm{Et}_{3} \mathrm{~N}-5 \mathrm{HF}$ 的存在下, 对甲基碘苯在阳极发生 氧化生成二氟碘苯，随后受到烯丙基酰胺双键的亲核进 攻生成碘鎓离子中间体，接着在羰基的作用下发生开环 生成三价碘中间体, 最后, 在亲核试剂 $\mathrm{F}^{-}$的进攻下发生 $\mathrm{S}_{\mathrm{N}} 2$ 取代生成目标产物.

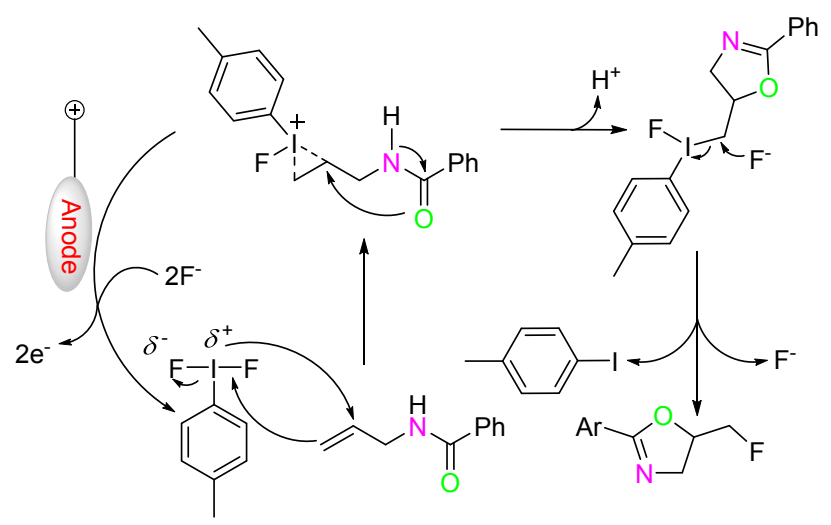

图式 23 氟盐为媒介电化学催化合成 5-氟甲基-2-噁唑啉类化 合物的可能机理

Scheme 23 Proposed reaction mechanism for electro-catalyzed synthesis of 5-fluoromethyl-2-azolines mediated by fluorine salt

随后, 该课题组 ${ }^{[31]}$ 报道了 $\mathrm{Et}_{3} \mathrm{~N}-5 \mathrm{HF}$ 存在下炔丙基 酰胺的氟环化反应(Scheme 24). 当碘苯为 2 equiv. 时, 电流密度 $50 \mathrm{~mA} / \mathrm{cm}^{2}$, 恒电流电解 $15 \mathrm{~h}$, 氟环化产物啞 唑产率为 $53 \%$. 在最优条件下，底物拓展结果显示，梄、 卤素、硝基、烷基、腈基等官能团均不受影响. 值得一

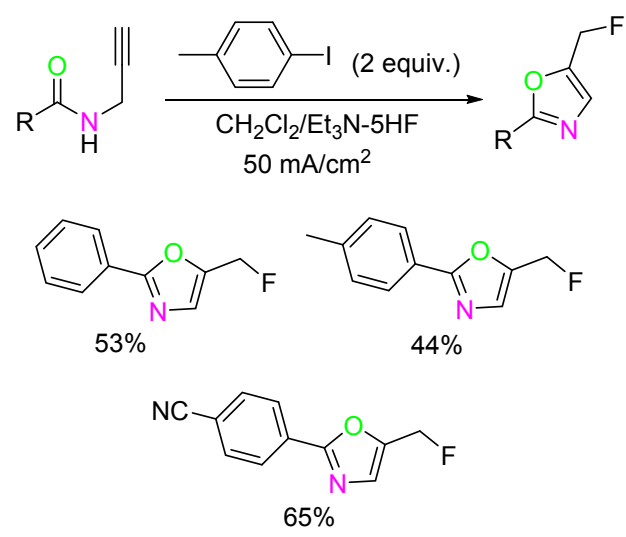

图式 24 电化学催化炔丙基酰胺的氟环化反应 Scheme 24 Eectro-catalyzed fuorocyclization of $N$-popargylamides 
提的是, 该反应可以通过调控碘苯的化学计量实现产物 的选择性.

\section{4 结论与展望}

相对于传统合成含氮杂环化合物的方法, 卤素盐参 与下，有机电合成该类化合物的入反应具有简单、高效、 绿色和经济等特点. 该类反应近几年的发展已经实现了 吲哚、咪唑、吡嗪等含氮杂环化合物的构建, 许多反应 展现出潜在的工业应用前景. 随着绿色化学和可持续发 展化学的新要求, 该类反应仍然存在未解决的问题和挑 战. 如反应往往需要额外加入电解质, 底物局限性和反 应立体选择性未解决等. 因此, 将来对这类反应的研究, 有必要寻找合适的卤素盐作媒介来得到高化学选择性 的产物. 研究探索多组分一锅法的串联反应来实现反应 步骤经济性. 随着越来越多的研究者加入到这个领域, 我们相信，卤素盐参与下有机电合成含氮杂环化合物将 在现代有机合成中发挥更大的作用.

\section{References}

[1] (a) Sabbasani, V. R.; Wang, K. P.; Streeter, M. D.; Spiegel, D. A. Angew. Chem. Int. Ed. 2019, 58, 18913.

(b) Draghici, C.; Wang, T.; Spiegel, D. A. Science 2015, 350, 294.

(c) Wang, S.; Huang, W.; Zhang, X.; Zhang, X.; Pan, C. Chin. J. Org. Chem. 2020, 40, 959 (in Chinese).

(王淑琴, 黄婉云, 张小蓉, 张晓婷, 潘成学, 有机化学, 2020, 40, 959.)

(d) Li, M.; Wang, R.; Hao, W.; Jiang, B. Chin. J. Org. Chem. 2020, 40, 1540 (in Chinese).

(李梦帆，王榕，郝文娟，姜波，有机化学, 2020, 40, 1540.)

(e) Zhang, F.; Peng, X.; Ma, J. Chin. J. Org. Chem. 2019, 39, 109 (in Chinese).

(张发光, 彭星, 马军安, 有机化学, 2019, 39, 109.)

(f) Sun, Y.; Zhou, L.; Wang, L. Chin. J. Org. Chem. 2019, 39, 3516 (in Chinese).

(孙悦玮, 周来运, 王兰芝, 有机化学, 2019, 39, 3516.)

(g) Meng, L.; Wang, Z.-Y. Chin. Chem. Lett. 2013, 24, 780.

[2] (a) Ye, Z.; Zhang, F. Chin. J. Chem. 2019, 37, 513.

(b) Liu, Z.; Zhang, Y.-S.; Wei, Y.; Shi, M. Eur. J. Org. Chem. 2020, 2020, 1093.

(c) Sundar, S.; Rengan, R. Org. Biomol. Chem. 2019, 17, 1402.

(d) Sridevi, B.; Kandimalla, S. R.; Reddy, B. V. S. Eur. J. Org. Chem. 2019, 2019, 6800.

(e) Rossi, R.; Angelici, G.; Casotti, G.; Manzini, C.; Lessi, M. Adv. Synth. Catal. 2019, 361, 2737.

(f) Roslan, I. I.; Ng, K.-H.; Jaenicke, S.; Chuah, G.-K. Catal. Sci. Technol. 2019, 9, 1528.

(g) Parvatkar, P. T.; Manetsch, R.; Banik, B. K. Chem. Asian J. 2019, 14, 6 .

(h) Salfeena, C. T. F.; Jalaja, R.; Davis, R.; Suresh, E.; Somappa, S. B. Acs Omega 2018, 3, 8074.

(i) Monga, A.; Bagchi, S.; Sharma, A. New J. Chem. 2018, 42, 1551.

(j) Felipe-Blanco, D.; Gonzalez-Gomez, J. C. Adv. Synth. Catal. 2018, 360, 2773.

(k) Donthiboina, K.; Namballa, H. K.; Shaik, S. P.; Nanubolu, J. B.;
Shankaraiah, N.; Kamal, A. Org. Biomol. Chem. 2018, 16, 1720.

(1) Chen, X.; Wang, Z.; Huang, H.; Deng, G.-J. Adv. Synth. Catal. 2018, 360, 4017

(m) Zhou, X.; Ma, H.; Shi, C.; Zhang, Y.; Liu, X.; Huang, G. Eur. J. Org. Chem. 2017, 2017, 237.

(n) Tjutrins, J.; Arndtsen, B. A. Chem. Sci. 2017, 8, 1002.

(o) Sunkari, S.; Shaik, S. P.; Krishna, N. H.; Rao, A. V. S.; Kodiripaka, B. G.; Alarifi, A.; Kamal, A. Asian J. Org. Chem. 2017, $6,1830$.

(p) Singh, D.; Kumar, V.; Devi, N.; Malakar, C. C.; Shankar, R.; Singh, V. Adv. Synth. Catal. 2017, 359, 1213.

(q) Saito, A. ARKIVOC 2017, 84

[3] (a) Leech, M. C.; Lam, K. Acc. Chem. Res. 2020, 53, 121

(b) Jiao, K.-J.; Xing, Y.-K.; Yang, Q.-L.; Qiu, H.; Mei, T.-S. Acc. Chem. Res. 2020, 53, 300.

(c) Yuan, Y.; Lei, A. Acc. Chem. Res. 2019, 52, 3309.

(d) Xiong, P.; Xu, H.-C. Acc. Chem. Res. 2019, 52, 3339.

(e) Fu, N.; Song, L.; Liu, J.; Shen, Y.; Siu, J. C.; Lin, S. J. Am. Chem. Soc. 2019, 141, 14480.

(f) Yan, M.; Kawamata, Y.; Baran, P. S. Chem. Rev. 2017, 117, 13230.

[4] (a) Xiong, P.; Xu, H.-H.; Xu, H.-C. J. Am. Chem. Soc. 2017, 139, 2956.

(b) Yang, Y.-Z.; Wu, Y.-C.; Song, R.-J.; Li, J.-H. Chem. Commun. 2020, 56, 7585 .

(c) Luo, M.-J.; Li, Y.; Ouyang, X.-H.; Li, J.-H.; He, D.-L. Chem. Commun. 2020, 56, 2707.

(d) Luo, M.-J.; Zhang, T.-T.; Cai, F.-J.; Li, J.-H.; He, D.-L. Chem. Commun. 2019, 55, 7251.

[5] Tang, H.-T.; Jia, J.-S.; Pan, Y.-M. Org. Biomol. Chem. 2020, 18, 5315.

[6] Chen, J.; Yan, W. Q.; Lam, C. M.; Zeng, C. C.; Hu, L. M.; Little, R. D. Org. Lett. 2015, 17, 986.

[7] Wang, H.; Shi, J.; Tan, J.; Xu, W.; Zhang, S.; Xu, K. Org. Lett. 2019, 21, 9430 .

[8] Ma, H.-Y.; Zha, Z.-G.; Zhang, Z.-L.; Meng, L.; Wang, Z.-Y. Chin. Chem. Lett. 2013, 24, 780.

[9] Wang, Z.-Q.; Meng, X.-J.; Li, Q.-Y.; Tang, H.-T.; Wang, H.-S.; Pan, Y.-M. Adv. Synth. Catal. 2018, 360, 4043.

[10] Mo, S.-K.; Teng, Q.-H.; Pan, Y.-M.; Tang, H.-T. Adv. Synth. Catal. 2019, 361, 1756

[11] Wang, H.; Zhang, J.; Tan, J.; Xin, L.; Li, Y.; Zhang, S.; Xu, K. Org. Lett. 2018, 20, 2505.

[12] Yang, N.; Lai, Q.; Jiang, H.; Yuan, G. Electrochem. Commun. 2016, $72,109$.

[13] Yang, N.; Yuan, G. J. Org. Chem. 2018, 83, 11963.

[14] Zeng, L.; Li, J.; Gao, J.; Huang, X.; Wang, W.; Zheng, X.; Gu, L.; Li, G.; Zhang, S.; He, Y. Green Chem. 2020, 22, 3416.

[15] Li, W.-C.; Zeng, C.-C.; Hu, L.-M.; Tian, H.-Y.; Little, R. D. Adv. Synth. Catal. 2013, 355, 2884.

[16] Gao, W.-J.; Li, W.-C.; Zeng, C.-C.; Tian, H.-Y.; Hu, L.-M.; Little, R. D. J. Org. Chem. 2014, 79, 9613.

[17] Tang, S.; Gao, X.; Lei, A. Chem. Commun. 2017, 53, 3354.

[18] Qian, P.; Yan, Z.; Zhou, Z.; Hu, K.; Wang, J.; Li, Z.; Zha, Z.; Wang, Z. Org. Lett. 2018, 20, 6359.

[19] Qian, P.; Yan, Z.; Zhou, Z.; Hu, K.; Wang, J.; Li, Z.; Zha, Z.; Wang, Z. J. Org. Chem. 2019, 84, 3148.

[20] Feng, M.-L.; Li, S.-Q.; He, H.-Z.; Xi, L.-Y.; Chen, S.-Y.; Yu, X.-Q. Green Chem. 2019, 21, 1619

[21] Qian, P.; Su, J. H.; Wang, Y.; Bi, M.; Zha, Z.; Wang, Z. J. Org. Chem. 2017, 82, 6434.

[22] Liang, S.; Zeng, C.-C.; Luo, X.-G.; Ren, F.-z.; Tian, H.-Y.; Sun, 
B.-G.; Little, R. D. Green Chem. 2016, 18, 2222.

[23] Liu, K.; Song, C.; Wu, J.; Deng, Y.; Tang, S.; Lei, A. Green Chem. 2019, 21, 765 .

[24] Lv, S.; Han, X.; Wang, J.-Y.; Zhou, M.; Wu, Y.; Ma, L.; Niu, L.; Gao, W.; Zhou, J.; Hu, W.; Cui, Y.; Chen, J. Angew. Chem. Int. Ed. 2020, $59,11583$.

[25] Jiang, Y.-Y.; Liang, S.; Zeng, C.-C.; Hu, L.-M.; Sun, B.-G. Green Chem. 2016, 18, 6311.

[26] Jiang, Y.-Y.; Dou, G.-Y.; Xu, K.; Zeng, C.-C. Org. Chem. Front. 2018, 5, 2573.
[27] Zhang, S.; Li, L.; Xue, M.; Zhang, R.; Xu, K.; Zeng, C. Org. Lett. 2018, 20, 3443.

[28] Nikolaienko, P.; Jentsch, M.; Kale, A. P.; Cai, Y.; Rueping, M. Chem.-Eur. J. 2019, 25, 7177.

[29] Li, Y.; Ye, Z.; Chen, N.; Chen, Z.; Zhang, F. Green Chem. 2019, 21, 4035.

[30] Haupt, J. D.; Berger, M.; Waldvogel, S. R. Org. Lett. 2019, 21, 242.

[31] Herszman, J. D.; Berger, M.; Waldvogel, S. R. Org. Lett. 2019, 21, 7893.

(Fan, Y.) 